\title{
Exponential Decay of Green's Functions for a Class of Long Range Hamiltonians
}

\author{
Wei-Min Wang \\ Department of Physics, Princeton University, Princeton, NJ 08544, USA
}

Received April 25, 1990; in revised form June 18, 1990

\begin{abstract}
We consider a class of long range Hamiltonians with diagonal disorder on $l^{2}(\mathbf{Z})$. For any ergodic potential $V$ with non-empty essential range, we prove the exponential decay of the Green's functions for energies in the essential range. If $V$ is independent identically distributed, we obtain the exponential decay of the Green's functions for all coupling constant $\lambda>0$. Moreover the Hamiltonian has only pure point spectrum.
\end{abstract}

\section{Introduction}

We consider a long range Hamiltonian with diagonal disorder. Let $(\Omega, P)$ be a probability space, $T^{j}(j \in \mathbf{Z})$ a one parameter group of ergodic measure preserving transformations acting on $\Omega ; f$ a measurable real-valued function on $\Omega$, such that the distribution $P^{f}$ of $f$ has no point component: $P\{\omega \mid f(\omega)=E\}=0$ for any $E$. Let the corresponding ergodic potential $V^{\omega}(j)=f\left(T^{j} \omega\right)$; we define $H^{\omega}$ on $l^{2}(\mathbf{Z})$ by:

$$
H^{\omega}=\tilde{\Delta}+\lambda V^{\omega},
$$

where $\tilde{\Delta}$ is a long-range finite difference Laplacian:

$$
\tilde{\Delta}(m, n)=\frac{\alpha^{-|m-n|+1}}{1-\alpha^{2}} \quad(\alpha>1),
$$

$\left(1 /\left(1-\alpha^{2}\right)\right.$ is just a normalizing factor), and $\lambda$ is the coupling constant. (We will write $V, H$, instead of $V^{\omega}, H^{\omega}$ for convenience.)

Clearly $\tilde{\Delta}$ is very different from the usual finite difference Laplacian $\Delta$ : $\Delta(m, n)=\delta(|m-n|-1)$, and is perhaps physically more realistic.

We are interested in the asymptotic behavior of the Green's functions for $H$. We show in the next section that $H$ can be written as

$$
H=\frac{1}{\Delta-\left(\alpha+\alpha^{-1}\right)}+\lambda V .
$$


Define $H_{\varepsilon}$ by

$$
H_{\varepsilon}=\frac{1}{\Delta-\left(\alpha+\alpha^{-1}+i \varepsilon\right)}+\lambda V .
$$

Note that $H=H_{\varepsilon}(\varepsilon=0)$. The Green's function for $E$ in the spectrum is defined to be

$$
G(m, n ; E)=\lim _{\varepsilon \rightarrow 0} G_{\varepsilon}(m, n ; E)=\lim _{\varepsilon \rightarrow 0}\left\langle\delta_{m}\left|\left(H_{\varepsilon}-E\right)^{-1}\right| \delta_{n}\right\rangle .
$$

It is easy to see that $\left(H_{\varepsilon}-E\right)^{-1}$ is bounded for $\varepsilon>0$. We prove below that the above limit exists for Lebesgue almost all $\alpha(L-$ a.a. $\alpha)$, and is in most cases the same as the more conventional definition: $G(m, n ; E)=\lim _{\varepsilon \rightarrow 0}\left\langle\delta_{m}\left|(H-E-i \varepsilon)^{-1}\right| \delta_{n}\right\rangle$.

Definition. $E$ is in the essential range (ER) of $f$ if and only if

$$
P\{\omega \mid E-\varepsilon<f(\omega)<E+\varepsilon\}>0
$$

for all $\varepsilon>0$.

The main results of the paper are as follows:

Theorem 1.1. For all $E, P$ - a.a. $\omega, L-$ a.a. $\alpha>1, G(m, n ; E)=\lim _{\varepsilon \rightarrow 0} G_{\varepsilon}(m, n ; E)$ exists. Suppose $E$ is in the essential range of $\lambda f$ for some $\lambda$, and $\mathbf{E} \log ^{+} 1 /|\lambda V-E|<\infty$, then for a.a. $\omega$ and fixed $n$,

$$
\lim _{|m| \rightarrow \infty} \frac{\log \left|G^{\omega}(m, n ; E)\right|}{|m|}=-\gamma_{E},
$$

where $\gamma_{E}>0$, for a.a. $\alpha$. Furthermore

$$
G(m, n ; E)=\lim _{\varepsilon \rightarrow 0}\left\langle\delta_{m}\left|(H-E-i \varepsilon)^{-1}\right| \delta_{n}\right\rangle \quad \text { a.a. } \alpha .
$$

Remark. Assume the ER of $f$ contains an interval containing 0 , then any $E$ is in the ER of $\lambda f$ for sufficiently large $\lambda$.

Theorem 1.2. If $V$ is independent identically distributed (i.i.d.) with absolutely continuous distribution, and $\mathbf{E} \log ^{+} 1 /|\lambda V-E|<\infty$, then for all $\lambda>0$, all $E, P$ - a.a. $\omega$ and fixed $n$, both (1.4a) and (1.4b) hold for a.a. $\alpha$. The Hamiltonian $H$ has only pure point spectrum.

Remark. The condition $\mathbf{E} \log ^{+} 1 /|\lambda V-E|<\infty$ in the above two theorems is a regularity condition that enables one to use the multiplicative ergodic theorem [11] to show the existence of the Lyapunov exponent (see the proof of Lemma 2.1).

The proofs of Theorems 1.1 and 1.2 are based on the identity (2.4) of the following section together with results of Kotani [8] and the trace-class method of Simon and Spencer [13]. The ergodicity of $V$ plays a crucial role in establishing the theorems. In fact it is essentially the only condition required of $V$. In particular, $V$ need not be non-deterministic in the sense of Kotani $[8,12]$. The nondeterministic condition excludes many interesting stochastic potentials that are intuitively random [1]. In this sense Theorem 1.1 is a generalization of the Kotani theorem, which proves the positivity of the Lyapunov exponent for non-deterministic potentials only $[8,12]$. It also complements a new result of 
Kotani, which proves the positivity of the Lyapunov exponent for potentials that take a finite set of values but are non-periodic [9]. Note that this theorem holds for potentials with empty ER, while Theorem 1.1 holds for potentials with non-empty ER.

The potentials $V$ studied here include for example, quasi-periodic potentials and potentials with a Gaussian distribution. In particular, for the quasi-periodic cosine potential, Theorem 1.1 implies the positivity of the Lyapunov exponent for all $\lambda>0$ and all $E \in(-\lambda, \lambda)$. This is quite surprising in view of the strong $\lambda$ dependence of the Lyapunov exponent if we replace $\widetilde{\Delta}$ with $\Delta$ [1]. Note that (1.4a) does not hold for periodic potentials, since such a potential has empty ER and is not ergodic.

Similar results can be obtained for

where

$$
\bar{H}=\bar{\Delta}+\lambda V,
$$

$$
\bar{\Delta}_{i j}= \begin{cases}f(|i-j|), & 0<|i-j|<l(l \text { any integer }) \\ 0, & \text { otherwise }\end{cases}
$$

when $V$ is non-deterministic, and will be written elsewhere.

\section{Proof of the Theorems}

The kinetic part of the Hamiltonian can be resummed exactly (as can be easily seen in the Fourier space). We have

so that,

$$
\tilde{\Delta}=\frac{1}{\Delta-\left(\alpha+\alpha^{-1}\right)}
$$

$$
H=\frac{1}{\Delta-\left(\alpha+\alpha^{-1}\right)}+\lambda V
$$

Note the denominator $\Delta-\left(\alpha+\alpha^{-1}\right)$ is never zero, since $\|\Delta\|=2$ and $\alpha \neq 1$. So the operator is well defined. (We use fractions whenever the numerator and the denominator commute.)

The Green's function $G$ for $H$ is related to the Green's function $G^{\prime}$ for another Hamiltonian $H^{\prime}$,

where

$$
H^{\prime}=\Delta+V^{\prime},
$$

$$
V^{\prime}=\frac{1}{\lambda V-E} \text {. }
$$

(Note that $V^{\prime}$ is also an ergodic potential.) This can be easily accomplished by using (1.2):

$$
\begin{aligned}
\left(H_{\varepsilon}-E\right)^{-1} & =\left(\frac{1}{\Delta-\left(\alpha+\alpha^{-1}+\mathrm{i} \varepsilon\right)}+\lambda V-E\right)^{-1} \\
& =\left(\Delta-\left(\alpha+\alpha^{-1}+i \varepsilon\right)\right)\left(H^{\prime}-\left(\alpha+\alpha^{-1}+i \varepsilon\right)\right)^{-1}(\lambda V-E)^{-1} .
\end{aligned}
$$


Let

$$
G^{\prime}\left(m, n ; \alpha+\alpha^{-1}+i \varepsilon\right)=\left\langle\delta_{m}\left|\left(H^{\prime}-\left(\alpha+\alpha^{-1}+i \varepsilon\right)\right)^{-1}\right| \delta_{n}\right\rangle
$$

then

$$
\begin{aligned}
G(m, n ; E)= & \lim _{\varepsilon \rightarrow 0}\left\langle\delta_{m}\left|\left(H_{\varepsilon}-E\right)^{-1}\right| \delta_{n}\right\rangle \\
= & \lim _{\varepsilon \rightarrow 0} \frac{1}{\lambda V_{n}-E}\left[G^{\prime}\left(m+1, n ; \alpha+\alpha^{-1}+i \varepsilon\right)+G^{\prime}\left(m-1, n ; \alpha+\alpha^{-1}+i \varepsilon\right)\right. \\
& \left.-\left(\alpha+\alpha^{-1}+i \varepsilon\right) G^{\prime}\left(m, n ; \alpha+\alpha^{-1}+i \varepsilon\right)\right] .
\end{aligned}
$$

Remarks. For $\varepsilon>0, G^{\prime}$ is well defined, since $H^{\prime}$ is self-adjoint. The Hamiltonian $H$ is self-adjoint, so $G(m, n)=G(n, m)$. Once we establish the exponential decay of $G(m, n ; E)$ for fixed $n$ and $|m| \rightarrow \infty$, the result also holds for fixed $m$ and $|n| \rightarrow \infty$. It is clear that in order to study $G(m, n ; E)$, it is equivalent to study $\lim _{\varepsilon \rightarrow 0} G^{\prime}\left(m, n ; \alpha+\alpha^{-1}+i \varepsilon\right)$.

The Lyapunov exponent $\gamma_{E}(\mathscr{E})$ characterizes the asymptotic behavior of the solutions to $H^{\prime} u=\mathscr{E} u$. If we denote the transfer matrices by $\left\{A_{n}\right\}$, then for each $\mathscr{E}$, the Lyapunov exponent for $H^{\prime}$ is defined to be [1]:

$$
\gamma_{E}(\mathscr{E})=\lim _{n \rightarrow \infty} \frac{\log \left\|A_{n} \cdots A_{1}\right\|}{n}, \text { a.a. } \omega \text {. }
$$

The above limit exists and is independent of $\omega$ for $P$ - a.a. $\omega$ [5].

Lemma 2.1. For a.a. $\alpha, \lim _{\varepsilon \rightarrow 0} G^{\prime}\left(m, n ; \alpha+\alpha^{-1}+i \varepsilon\right)$ exists. If furthermore $\gamma_{E}\left(\alpha+\alpha^{-1}\right)>0$ for a.a. $\alpha$, then

$$
\lim _{|m| \rightarrow \infty} \frac{1}{|m|} \log \lim _{\varepsilon \rightarrow 0}\left|G^{\prime}\left(m, n ; \alpha+\alpha^{-1}+i \varepsilon\right)\right|=-\gamma_{E}\left(\alpha+\alpha^{-1}\right), \quad \text { a.a. } \alpha .
$$

Proof. Since $G^{\prime}\left(m, m ; \alpha+\alpha^{-1}+i \varepsilon\right)$ is a Herglotz function, for a.a. $\alpha, \lim _{\varepsilon \rightarrow 0} G^{\prime}(m, m)$ exists [7,8]; $\lim _{\varepsilon \rightarrow 0} G^{\prime}(m, n)(m \neq n)$ exists by polarization. Using the multiplicative ergodic theorem and an argument due to Delyon, Lévy and Souillard [3] which shows that the Green's function decays at the rate of the Lyapunov exponent, we obtain (2.8).

The positivity of the Lyapunov exponent is ensured by the following two lemmas:

Lemma 2.2. For $E$ in the ER of $\lambda f$, define $\Omega^{\prime}$ to be the set of $\omega$, such that there exists a subsequence $j_{n}$, with $j_{n} \rightarrow \pm \infty$, such that $\lim _{n \rightarrow \infty} \lambda V_{j_{n}}(\omega)=E$, and $\lambda V_{j}(\omega) \neq E$, for all $j$.

Then the set $\Omega^{\prime}$ has full measure: $P\left(\Omega^{\prime}\right)=1$.

The main lemma is:

Lemma 2.3. If $E$ is in the $\mathrm{ER}$ of $\lambda f$, then for $\omega \in \Omega^{\prime}$ the Lyapunov exponent for $H^{\prime}$ is positive: $\gamma_{E}\left(\alpha+\alpha^{-1}\right)>0$ a.a. $\alpha$.

Proof of Lemma 2.2. Define $\Omega_{\varepsilon}$ to be set of $\omega$, such that there exists a subsequence 
$m_{i}, m_{i} \rightarrow \pm \infty$, such that $f\left(T^{m_{i}} \omega\right) \in(E / \lambda-\varepsilon, E / \lambda+\varepsilon)$. Clearly $\Omega_{\varepsilon}$ is $T$ invariant. Let $F_{\varepsilon}=f^{-1}(E / \lambda-\varepsilon, E / \lambda+\varepsilon)$, and $\chi_{F_{\varepsilon}}$ be the characteristic function of $F_{\varepsilon}$. If $\omega \notin \Omega_{\varepsilon}$, then

$$
\lim _{n \rightarrow \infty} \frac{1}{n} \sum_{m=0}^{m= \pm n} \chi\left(T^{m} \omega\right)=0
$$

but

$$
\int \chi(\omega) d P(\omega)>0
$$

since $E$ is in the ER of $\lambda f$. So $P\left(\Omega_{\varepsilon}\right)=1$. Define $\Omega^{0}$ to be,

$$
\Omega^{0}=\bigcap_{p=1}^{\infty} \Omega_{1 / p}
$$

then $P\left(\Omega^{0}\right)=1$. It is easy to see that the set

$$
\Omega^{0} \cap\left\{\omega \mid f\left(T^{n} \omega\right) \neq E, \forall n\right\},
$$

which is of full measure (since $P^{f}$ has no point component) is contained in $\Omega^{\prime}$. Therefore $P\left(\Omega^{\prime}\right)=1$.

To prove the remaining two lemmas, we need the following two theorems:

Theorem 2.4 (Simon-Spencer) [13]. Let $h$ be the operator,

$$
h=\Delta+V
$$

on $l^{2}(\mathbf{Z})$. Suppose that

$$
\limsup _{n \rightarrow \pm \infty}|V(n)|=\infty
$$

then $\sigma_{\mathrm{ac}}(h)=\varnothing$.

Define the essential closure for a set $A$, to be

$$
\bar{A}^{\text {ess }}=\{\kappa|| A \cap(\kappa-\varepsilon, \kappa+\varepsilon) \mid>0, \text { for all } \varepsilon>0\},
$$

where $\|$ denotes the Lesbesgue measure. We have

Theorem 2.5 (Ishii-Pastur-Kotani) [1,8].

$$
\sigma_{\text {ac }}\left(h_{\omega}\right)=\overline{\{\mathscr{E} \mid \gamma(\mathscr{E})=0}^{\text {ess }} .
$$

Proof of Lemma 2.3. Since $E$ is in the ER of $\lambda f$, from Lemma 2.2, for $\omega \in \Omega^{\prime}$ the potential $V^{\prime}$ as defined in (2.3a) is unbounded: $\limsup _{n \rightarrow \pm \infty}\left|V^{\prime}(n)\right|=\infty$. Applying Theorem 2.4, we obtain $\sigma_{\mathrm{ac}}\left(H^{\prime}\right)=\varnothing$. This in turn implies, by Theorem 2.5 , for $\omega \in \Omega^{\prime}, \gamma_{E}\left(\alpha+\alpha^{-1}\right)>0$ for a.a. $\alpha$.

We need one more lemma before proceeding to the proofs of Theorems 1.1 and 1.2.

Lemma 2.4. For all $\omega \in \Omega^{\prime}, \gamma_{E}\left(\alpha+\alpha^{-1}+i \varepsilon\right) \geqq \gamma_{E}\left(\alpha+\alpha^{-1}\right)$ a.a. $\alpha$. If $E$ is in the ER of $\lambda f$, then

$$
\lim _{|m| \rightarrow \infty} \frac{1}{|m|} \log \left|G^{\prime}\left(m, n ; \alpha+\alpha^{-1}+i \varepsilon\right)\right|=-\gamma_{E}\left(\alpha+\alpha^{-1}+i \varepsilon\right) \leqq-\gamma_{E}\left(\alpha+\alpha^{-1}\right)<0 .
$$


Proof. From the Thouless formula $[1,16]$, the Lyapunov exponent is related to the density of states as follows,

$$
\gamma_{E}(\mathscr{E})=\int \log |\mathscr{E}-x| d \rho_{E}(x)
$$

where $\mathscr{E}$ is a complex number and $d \rho_{E}(x)$ the density of states. Suppose $\mathscr{E}=\alpha+\alpha^{-1}+i \varepsilon$, then taking derivatives with respect to $\varepsilon$, we get [6],

$$
\begin{aligned}
\frac{d \gamma_{E}}{d \varepsilon}\left(\alpha+\alpha^{-1}+i \varepsilon\right) & =\operatorname{Im} \int \frac{d \rho_{E}(x)}{x-\left(\alpha+\alpha^{-1}+i \varepsilon\right)} \\
& =\mathbf{E}\left[\operatorname{Im} G^{\prime}\left(0,0 ; \alpha+\alpha^{-1}+i \varepsilon\right)\right] .
\end{aligned}
$$

Since $\int \frac{d \rho_{E}(x)}{x-z}$ is a Herglotz function in the upper half plane, $\lim _{\varepsilon \rightarrow 0} \frac{d \gamma_{E}}{d \varepsilon}$ exists for a.a. $\alpha ;(1 / \pi) \lim _{\varepsilon \rightarrow 0} \mathbf{E}\left(\operatorname{Im} G^{\prime}\right)$ is the density of states, so we have

$$
\lim _{\varepsilon \rightarrow 0} \frac{d \gamma_{E}}{d \varepsilon} \geqq 0
$$

for a.a $\alpha$. Hence $\gamma_{E}\left(\alpha+\alpha^{-1}+i \varepsilon\right) \geqq \gamma_{E}\left(\alpha+\alpha^{-1}\right)$, for a.a. $\alpha$. Applying Lemma 2.3, we obtain $\gamma_{E}\left(\alpha+\alpha^{-1}+i \varepsilon\right)>0$, for a.a. $\alpha$. Therefore for $P-$ a.a. $\omega$ there exist unique solutions $u_{ \pm}$to $H^{\prime}$ that decay exponentially at $\pm \infty[8,12]$. The Green's function $G^{\prime}$ constructed from the solutions $u_{ \pm}$decay exponentially at the rate of the Lyapunov exponent. We thus obtain (2.10).

We are now ready to prove Theorems 1.1 and 1.2.

Proof of Theorem 1.1. Let $\omega \in \Omega^{\prime}$; using Lemmas 2.1 and 2.3, we see that in the limit $\varepsilon \rightarrow 0$, the sum of the Green's functions $G^{\prime}$ in (2.6) exists and is of $O\left(e^{-\gamma_{E}|m|}\right)$. Since for $\omega \in \Omega^{\prime}$ and fixed $n, 1 /\left(\lambda V_{n}-E\right)$ is just a constant,

$$
\lim _{|m| \rightarrow \infty} \frac{\log |G(m, n ; E)|}{|m|}=-\gamma_{E}\left(\alpha+\alpha^{-1}\right)
$$

where $\gamma_{E}\left(\alpha+\alpha^{-1}\right)>0$, a.a. $\alpha$. Let

$$
\mathscr{G}_{\varepsilon}(m, n ; E)=\left\langle\delta_{m}\left|(H-E-i \varepsilon)^{-1}\right| \delta_{n}\right\rangle .
$$

Since we only have the bound $\left|\mathscr{G}_{\varepsilon}\right| \leqq 1 / \varepsilon$, we need to expand the resolvent identity to second order:

where

$$
\mathscr{G}_{\varepsilon}-G_{\varepsilon}=G_{\varepsilon} \Gamma G_{\varepsilon}+G_{\varepsilon} \Gamma G_{\varepsilon} \Gamma \mathscr{G}_{\varepsilon}
$$

$$
\begin{aligned}
& \Gamma=i \varepsilon(A-1), \\
& A=\frac{1}{\left(\Delta-\left(\alpha+\alpha^{-1}+i \varepsilon\right)\right)\left(\Delta-\left(\alpha+\alpha^{-1}\right)\right)} .
\end{aligned}
$$

Clearly $A$ is bounded, so $\|\Gamma\|=O(\varepsilon)$ as $\varepsilon \rightarrow 0$. Since $\left|\mathscr{G}_{\varepsilon}\right| \leqq 1 / \varepsilon$ and $G_{\varepsilon}(m, n)$ decays exponentially with a rate that is uniformly bounded below from Lemmas 2.4 and (2.6), the right-hand side of (2.15) converges uniformly in $\varepsilon$. We obtain (1.4b) by taking the limit. 
Proof of Theorem 1.2. Since $V$ i.i.d., $V^{\prime}$ is also i.i.d.. By a standard integral equation method, $\gamma_{E}\left(\alpha+\alpha^{-1}\right)>0$ for a.a. $\alpha[2,15]$. So (1.4a) holds by Lemmas 2.3 and 2.1, (1.4b) holds by the same argument as in Theorem 1.1. By (1.4a) and Fubini's theorem,

$$
\lim _{\varepsilon \rightarrow 0} \sum_{m}|G(m, n ; E+i \varepsilon)|^{2}<\infty
$$

for a.a. $\omega$, a.a. $\alpha$ and a.a. E. Hence there exists only pure point spectrum $[4,14]$.

Acknowledgements. I thank Tom Spencer for suggesting this problem and for his guidance. I thank Percy Deift for a critical reading of the manuscript and helpful discussions. I also thank Martin Andler, Henrique von Dreifus and Avram Soffer for useful conversations.

\section{References}

1. Cycon, H. L., Froese, R. G., Kirsch, W., Simon, B.: Schrödinger Operators. Berlin, Heidelberg, New York: Springer 1987

2. Delyon, F., Kunz, H., Souillard, B.: One dimensional wave equations in disordered media. J. Phys. A. 16, 25 (1983)

3. Delyon, F., Lévy, Y., Souillard, B.: Anderson localization for one and quasi-one dimensional systems. J. Stat. Phys. 41, 375-388 (1985)

4. Delyon, F. Lévy, Y., Souillard, B.: Anderson localization for multidimensional systems at large disorder or large energy. Commun. Math. Phys. 100, 463 (1985)

5. Furstenberg, H., Kesten, H.: Products of random matrices. Ann. Math. Stat. 31, 457-469 (1960)

6. Johnson, R., Moser, J.: The rotation number for almost periodic potentials. Commun. Math. Phys. 84, 403-438 (1982)

7. Katznelson, Y.: An introduction to Harmonic analysis. New York: Dover 1976

8. Kotani, S.: Lyapunov indices determine absolutely continuous spectra of stationary random one dimensional Schrödinger operators. Proc. Kyoto Stoch. Conf. 225-248 (1982)

9. Kotani, S.: Jacobi matrices with random potentials taking finitely many values. Rev. Math. Phys. 1, 129-133 (1989)

10. Kunz, H., Souillard, B.: Sur le spectre des opérateurs aux différences finies aléatoires. Commun. Math. Phys. 78, 201 (1980)

11. Ruelle, D.: Erogdic theory of differentiable dynamical systems. IHES 50, 275 (1979)

12. Simon, B.: Kotani theory for one-dimensional stochastic Jacobi matrices. Commun. Math. Phys. 89, 227 (1983)

13. Simon, B., Spencer, T.: Trace class perturbations and the absence of absolutely continuous spectra. Commun. Math. Phys. 125, 113 (1989)

14. Simon, B., Wolff, T.: Singular continuous spectrum under rank one perturbations and localization for random Hamiltonians. Commun. Pure. Appl. Math 39, 75-90 (1986)

15. Spencer, T.: The Schrödinger equation with a random potential - a mathematical review. Critical Phenomena, Random Systems, Gauge Theories, Les Houches XLIII. Osterwalder, K., Stora, R. (eds.)

16. Thouless, D. J.: A relation between the density of states and range of localization for one dimensional random systems. J. Phys. C5, 77-81 (1972) 
